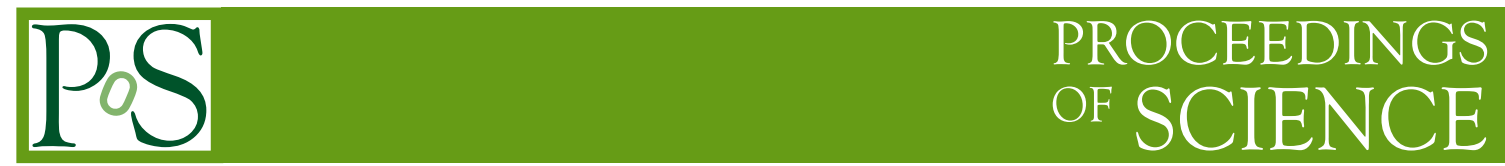

\title{
CMS results on the physics of top quarks
}

\author{
James Keaveney* \\ FWO Pegasus Marie Curie Fellow, Vrije Universiteit Brussel \\ E-mail: james.keaveney@vub.ac.be
}

The CMS top quark physics program consists of measurements of the cross sections of processes involving top quarks, the top-quark mass, and the properties of the top quark. The analyses described in this note utilise data recorded by CMS at centre-of-mass energies of 7 and $8 \mathrm{TeV}$ between the years 2010 and 2012. All results are consistent with standard model expectations.

The XXI International Workshop High Energy Physics and Quantum Field Theory,

June 23 - June 30, 2013

Saint Petersburg Area, Russia

${ }^{*}$ Speaker. 


\section{Introduction}

Measurements in the top quark sector form a central part of the CMS physics program. In this note a selection of these measurements are summarised. The analyses utilise data with centre-ofmass energies of 7 and $8 \mathrm{TeV}$ recorded by CMS between the years 2010 and 2012. The results are grouped into three categories: measurements of the cross sections of processes producing a $t \bar{t}$ system, measurements of the top-quark mass, and measurements of the properties of the top quark. Details of CMS results on single-top quark production can be found in [1]. Details of additional analyses in the CMS top quark physics program not described here can be found in [2].

\section{Measurements of $t \bar{t}$ cross sections}

\subsection{Inclusive $t \bar{t}$ cross sections}

Measuring the cross section of $t \bar{t}$ production $\left(\sigma_{t \bar{t}}\right)$ can provide a precise test of perturbative QCD (pQCD). Furthermore, as $t \bar{t}$ production is a dominant background for numerous searches for new physics phenomena at the LHC, a precise measurement of $\sigma_{t \bar{t}}$ can enhance the sensitivity of these searches. Finally, $t \bar{t}$ events provide a means of measuring crucial experimental parameters such as the Jet Energy Scale (JES) and the efficiency of identifying jets arising from $b$ quarks (b-tagging efficiency).

CMS measures $\sigma_{t \bar{t}}$ in both the single-lepton and dilepton decay channels using $2.8 \mathrm{fb}^{-1}$ and $2.4 \mathrm{fb}^{-1}$ of the $8 \mathrm{TeV}$ dataset respectively [3],[4]. In the single-lepton channel events are required to contain a single isolated muon or electron and four jets, one of which has been identified as originating from a $\mathrm{b}$ quark. To extract the cross section, a template fit to the distribution of the invariant mass of the lepton and b-jet system $\left(M_{l b}\right)$ is performed. In figure 1 the $M_{l b}$ distribution for data and simulation for the single-muon channel is shown. The measured cross section is: $\sigma_{t \bar{t}}=228.4 \pm 9.0(\text { stat } .)_{-26.0}^{+29.0}($ sys. $) \pm 10.0$ (lum. $)$ pb. The systematic uncertainty is dominated by the imperfect knowledge of the JES and b-tagging efficiency. In the dilepton channel, events are required to contain two leptons (electrons or muons) and a b-tagged jet. Due to the small backgrounds the cross section is extracted with a cut and count methodology. The measured cross section is $227 \pm 3$ (stat.) \pm 11 (syst.) \pm 10 (lumi.) pb. These results are mutually consistent and are in agreement with approximate next-to-next-to leading order $\mathrm{PQCD}$ predictions.

\subsection{Differential $t \bar{t}$ cross sections}

CMS measures $\sigma_{t \bar{t}}$ as a function of the final state kinematics using $12.2 \mathrm{fb}^{-1}$ of $8 \mathrm{TeV}$ data [5]. CMS also measure the $\sigma_{t \bar{t}}$ as a function of the jet multiplicity for various jet $\mathrm{P}_{T}$ requirements [8]. In both cases events are required to contain two leptons (electrons or muons) and a b-tagged jet. In figure 2 the distributions of the measured $\sigma_{t \bar{t}}$ as function of top-quark $P_{T}$ and jet multiplicity for jets with $P_{T}>60 \mathrm{GeV}$ are shown with predictions from various simulations included. For the top-quark $P_{T}$, good agreement is observed between data and the various predictions, although the measured spectrum exhibits a small excess (deficit) at low (high) top-quark $P_{T}$ relative to the simulation. The measured jet multiplicity distributions in $t \bar{t}$ events are reasonably well described by the various generators shown. Differential measurements of $\sigma_{t \bar{t}}$ are also available in the lepton plus 

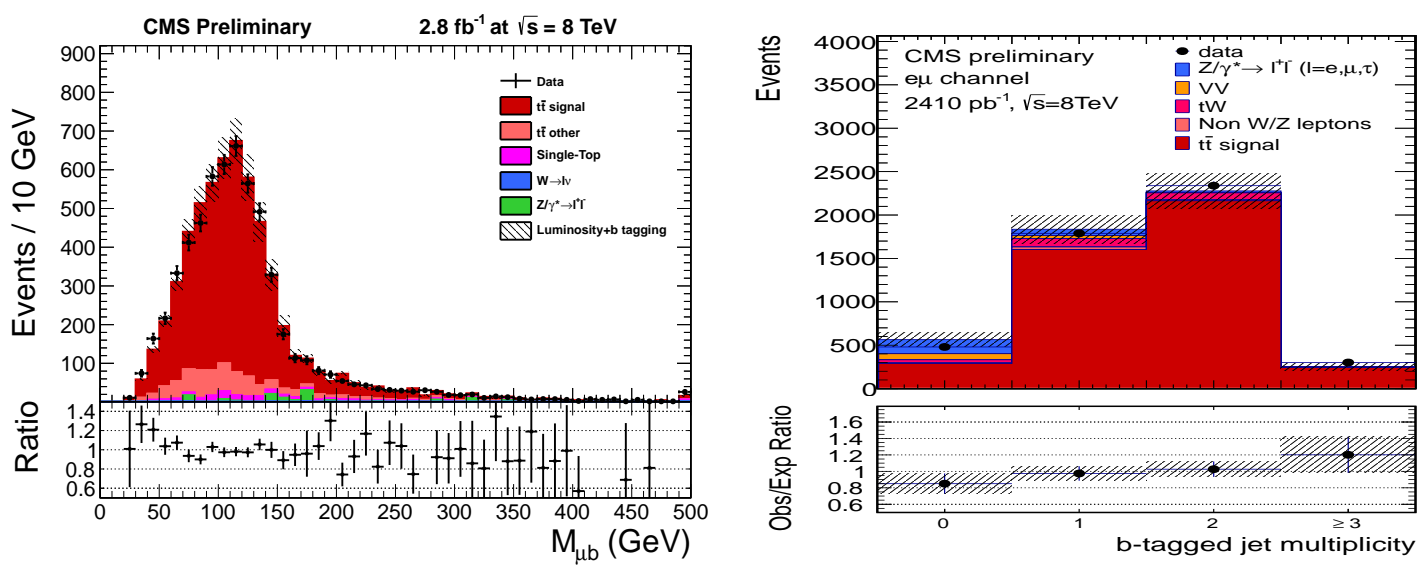

Figure 1: The $M_{\mu b}$ distribution in the single muon channel (left) and the b-tagged jet multiplicity distribution in the $e \mu$ channel (right) for data and simulation are shown.

jets channel [6] and at centre-of-mass energy of $7 \mathrm{TeV}$ [7]. A measurement of the jet multiplicity in $t \bar{t}$ events is also available at centre-of-mass-energy of $7 \mathrm{TeV}$ [9].
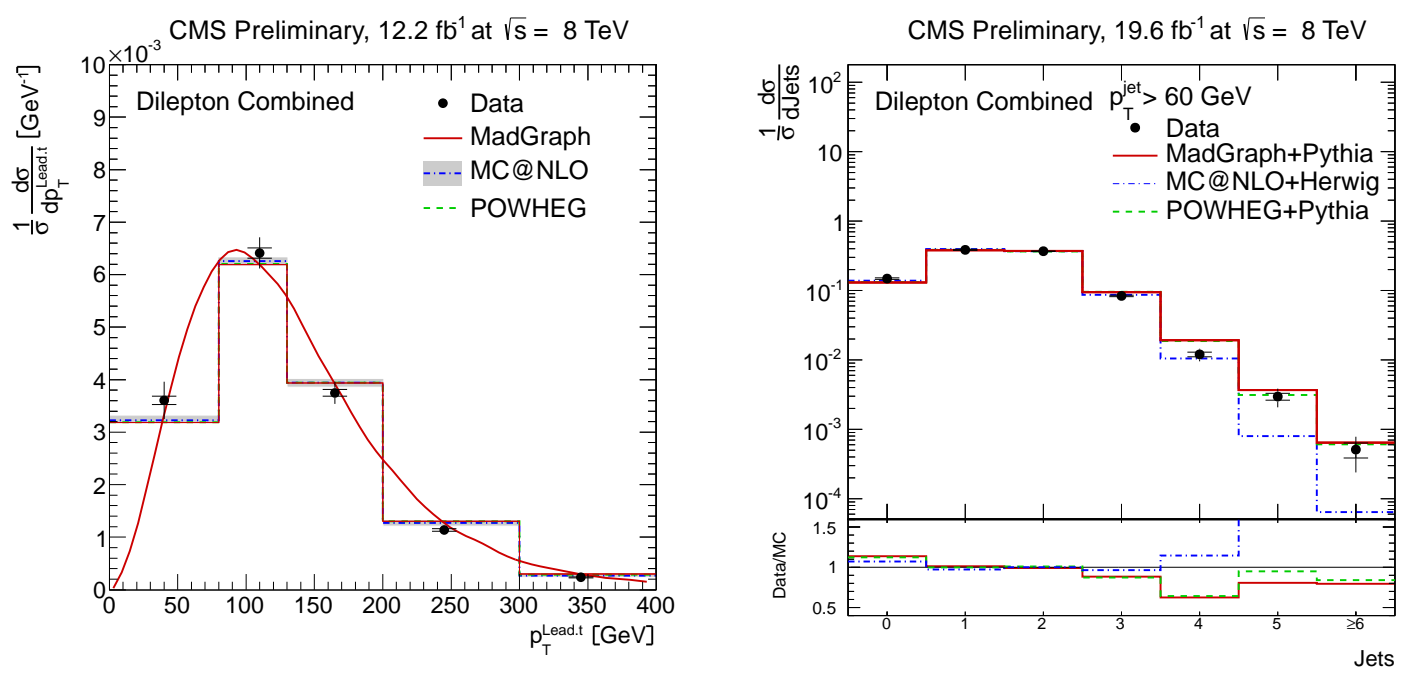

Figure 2: The $\sigma_{t \bar{t}}$ as a function of top-quark $P_{T}$ (left) and jet multiplicity distribution (right) in the dilepton channel for data and various simulations are shown.

\section{$2.3 \frac{\sigma_{t \bar{t}+b b}}{\sigma_{t \bar{t}+j \bar{j}}}$ cross section ratio}

The production of $t \bar{t}$ in association with a pair or bottom quarks $(b \bar{b})$ is an irreducible background to the production of $t \bar{t}$ in association with a Higgs boson $(t \bar{t} H)$ where the Higgs boson decays to a pair of bottom quarks. Hence a precise measurement of $\sigma_{t \bar{t}+b b}$ has the potential to reduce background uncertainty and thus increase sensitivity in searches for $t \bar{t} H$. CMS measures the cross section $\frac{\sigma_{t \bar{t}+b b}}{\sigma_{t \bar{t}+j \bar{j}}}$ where $t \bar{t}+j \bar{j}$ is defined as the production of a $t \bar{t}$ system in association with two jets of any flavour. The analysis uses the full $\left(5.0 \mathrm{fb}^{-1}\right) 7 \mathrm{TeV}$ dataset [10]. Events are required 
to contain two leptons (electrons or muons) and four jets, two of which have been b-tagged. The measurement is performed via a fit to the b-tagged jet multiplicity distribution and is corrected to particle level. In figure 3 the b-tagged jet multiplicity distributions for all channels are shown. The result is $\frac{\sigma_{t \bar{t}+b b}}{\sigma_{t \bar{t}+j \bar{j}}}=3.6 \pm 1.1$ (stat.) \pm 0.9 (syst.) $\%$.

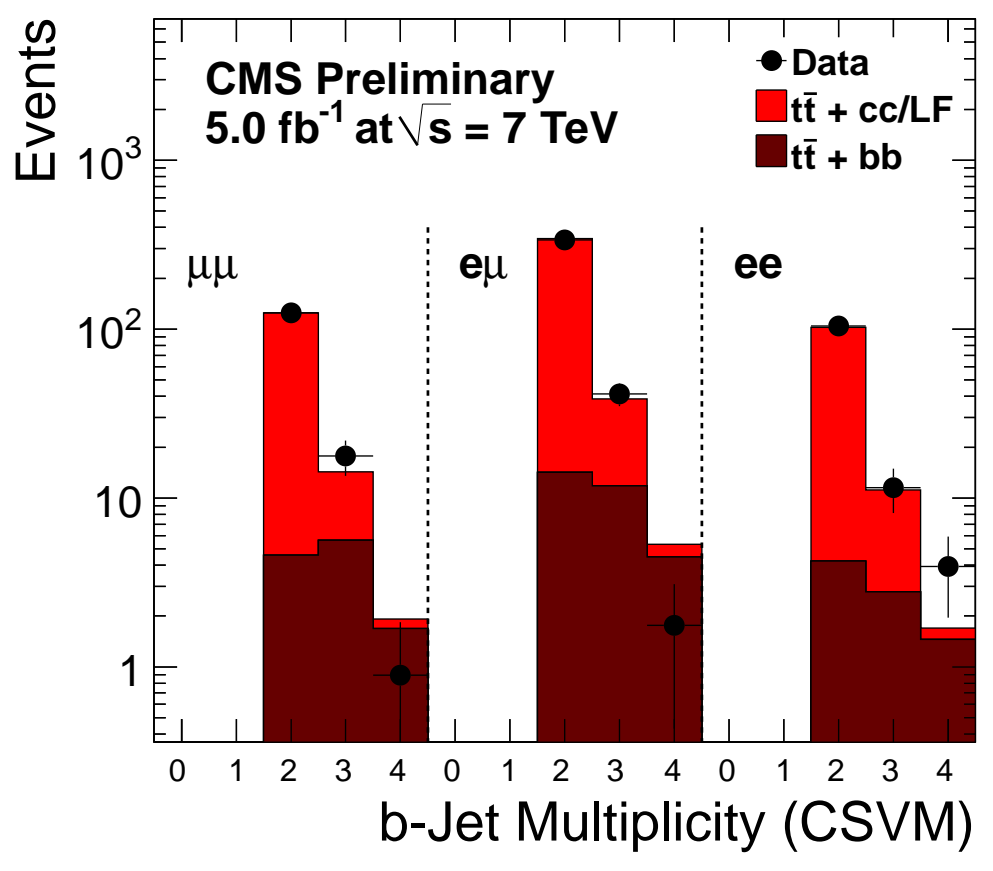

Figure 3: The distribution of the multiplicity of b-tagged jets is shown for data and simulation.

\section{$2.4 t \bar{t}+\mathbf{V}$ cross sections}

A measurement of the production of a $t \bar{t}$ system in association with a vector boson $(t \bar{t}+\mathrm{V})$ is a key test of the validity of the $\mathrm{SM}$ at the $\mathrm{TeV}$ scale. CMS performs the first measurement of $\sigma_{t \bar{t}+V}$ [11] using the full $7 \mathrm{TeV}$ dataset. Two complementary analyses are employed: one based on the trilepton signatures produced in $t \bar{t} Z$ decays and one based on same-sign dilepton signatures produced by $t \bar{t}+\mathrm{V}$ signatures where $\mathrm{V}=\mathrm{W}$ or $\mathrm{Z}$. The trilepton analysis aims to select events from the process $p p \rightarrow t \bar{t} Z \rightarrow\left(t \rightarrow b l^{ \pm} v\right)(t \rightarrow b j j)\left(Z \rightarrow l^{ \pm} l^{ \pm}\right)$. The dilepton analysis aims to select events originating from the processes: $p p \rightarrow t \bar{t} W \rightarrow\left(t \rightarrow b l^{ \pm} v\right)(t \rightarrow b j j)\left(W \rightarrow l^{ \pm} v\right)$ and $p p \rightarrow t \bar{t} Z \rightarrow\left(t \rightarrow b l^{ \pm} v\right)(t \rightarrow b j j)\left(Z \rightarrow l^{ \pm} l^{ \pm}\right)$(with $l=e$ or $\left.\mu\right)$.

In Fig. 4 the event yields separated in lepton flavour channels for the trilepton and dilepton analyses are shown. A total of 16 events are selected in data, compared to an expected background contribution of $9.2 \pm 2.6$ events. The significance of the observed $t \bar{t} V$ signal is equivalent to 3.0 standard deviations. A combined cross section is measured simultaneously from the three channels with the result: $\sigma_{t \bar{t} V}=0.43_{-0.15}^{+0.17}(\text { stat } .)_{-0.07}^{+0.09}($ sys. $) \mathrm{pb}$. This result is compatible with the NLO prediction of the standard model which is $\sigma_{t \bar{V} V}^{S M}=0.306_{-0.053}^{+0.031} \mathrm{pb}$. 

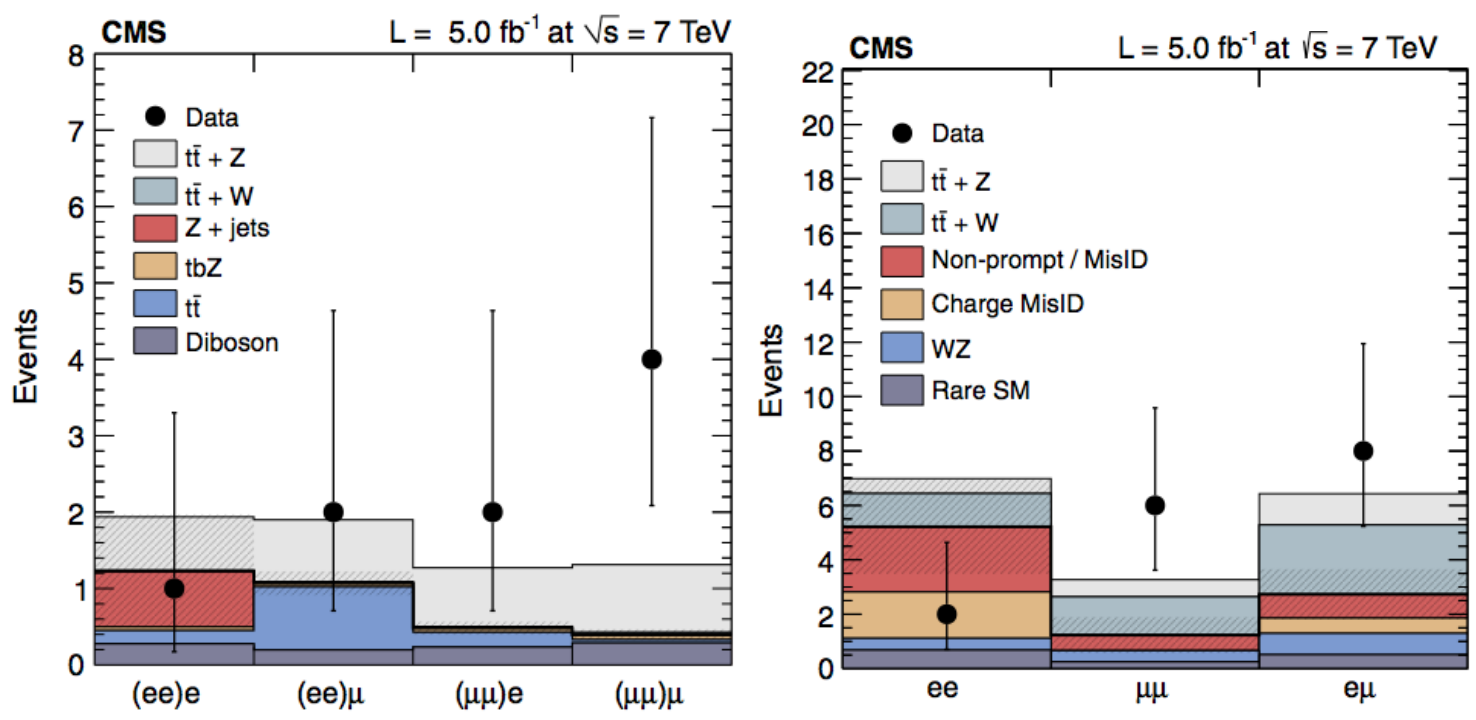

Figure 4: Event yields separated in lepton flavour channels for the trilepton (left) and dilepton channels (right). The expected contributions from signal and background processes are indicated.

\section{Measurements of the top-quark mass}

\subsection{Top-quark mass}

The top-quark mass $\left(m_{t}\right)$ is an essential parameter of the standard model. In addition, a precise measurement of $m_{t}$ also provides an important benchmark for the performance and calibration of the CMS detector. CMS measures $m_{t}$ using events containing a single lepton (electron or muon) and four jets, two of which have been b-tagged with the full $7 \mathrm{TeV}$ dataset [12]. A kinematic fit of jet combinations in the event is employed to check the compatibility of an event with the $t \bar{t}$ hypothesis and thereby improve the resolution of the measured quantities. To suppress the effect of incorrect combinations fit solutions are weighted by their fit probability. Finally a two-dimensional maximum likelihood fit is performed to extract the best fit value of $m_{t}$ and the JES. In figure 5 the invariant mass distribution of the reconstructed top quarks and a two-dimensional plot of the likelihood as a function of $m_{t}$ and the JES are shown. The top-quark mass is measured to be $m_{t}=173.49 \pm 0.43$ (stat. $\left.+J E S\right) \pm 0.98$ (syst.) GeV.

\subsection{Dependence of of top-quark mass on event kinematics}

CMS measures $m_{t}$ as a function of the event kinematics [13]. This is the first data measurement of possible kinematic biases in the measurements of $m_{t}$. The analysis uses a sample of candidate $t \bar{t}$ events with one electron or muon and at least four jets in the final state, collected by CMS at $\sqrt{s}=7 \mathrm{TeV}$. Similarly to [12], for each event the mass is reconstructed from a kinematic fit of the decay products to a $t \bar{t}$ hypothesis. The top-quark mass is determined simultaneously with the JES, constrained by the known mass of the $\mathrm{W}$ boson in $q \bar{q}$ decays. The measured values of $m_{t}$ are binned in various kinematic variables and the dependencies of $m_{t}$ on these variables is compared to those predicted by the MADGRAPH [14], POWHEG [15], [16] and MC@ NLO [17] simulations. In fig 6, the measured values $m_{t}$ in bins of the $P_{T}$ of the hadronically decaying top quark and the 

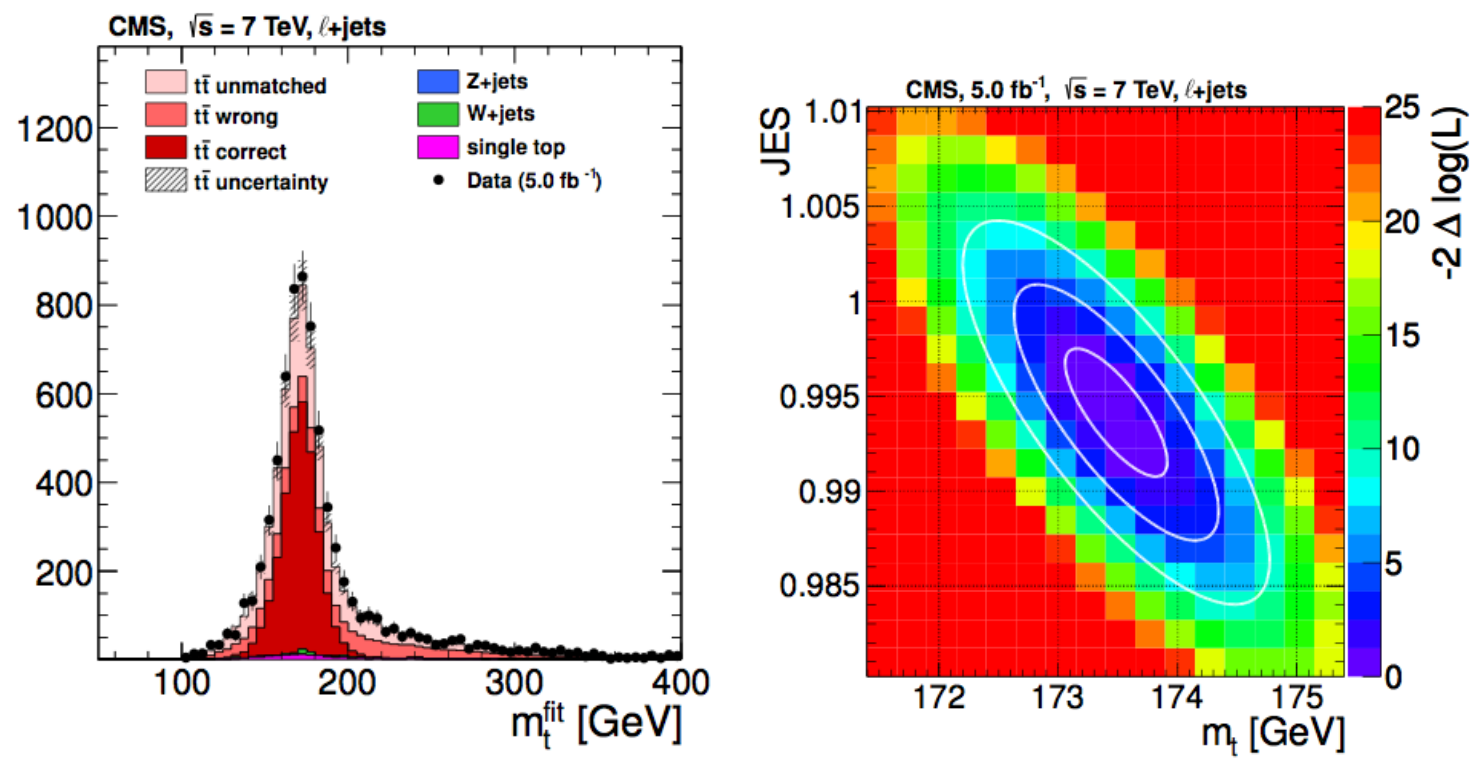

Figure 5: The invariant mass distribution of the reconstructed top quarks (left) and the two-dimensional likelihood (right) are shown. The ellipses on the likelihood plot correspond to statistical uncertainties on $m_{t}$ and JES of one, two, and three standard deviations.

$H_{T}$, where $H_{T}$ is the scalar sum of the $P_{T}$ of the selected jets in the event, are shown. For all the kinematic variables considered the observed dependencies are well reproduced by the simulation with tunes including and excluding the effects of colour reconnection.
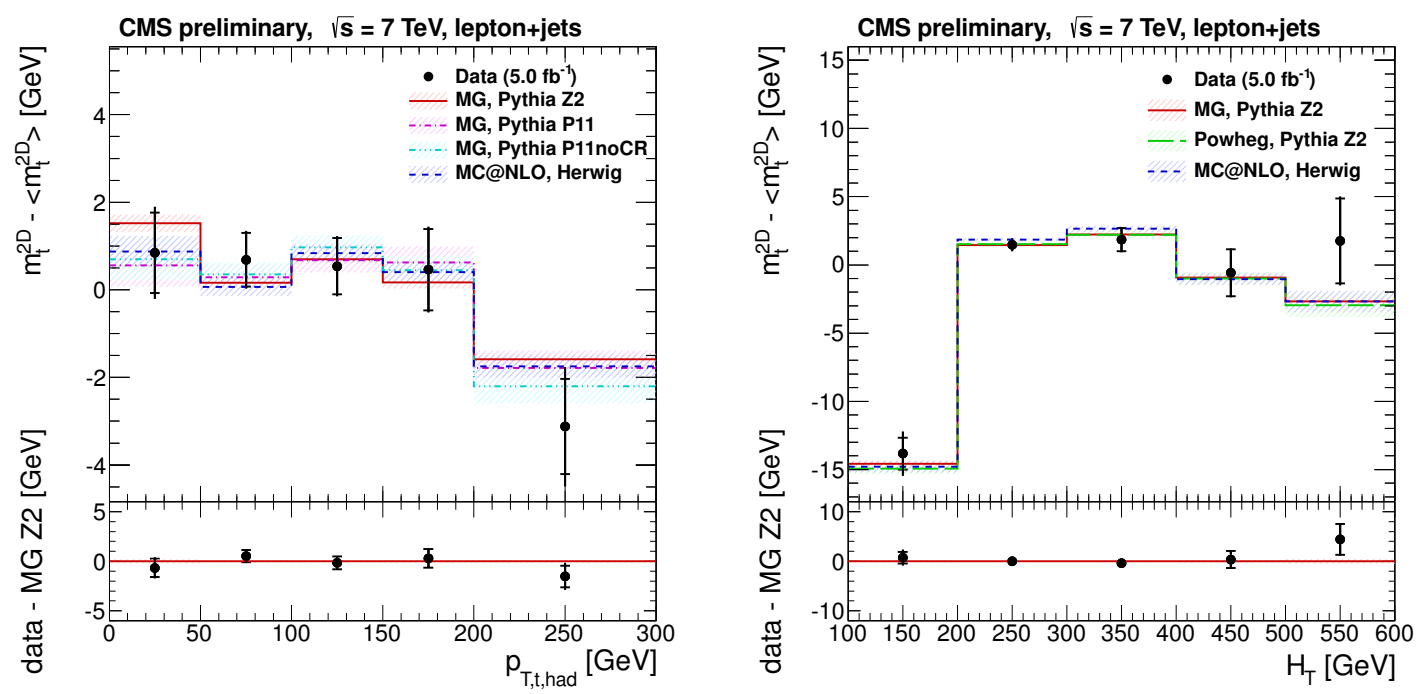

Figure 6: The measured values of $m_{t}$ in bins of the $P_{T}$ of the hadronically decaying top quark and $H_{T}$ are shown.

\subsection{Top-quark mass difference}

The invariance of the standard model under the CPT transformation implies the equality of 
particle and antiparticle masses. Hence a measurement of the mass difference between top and antitop quarks $\left(\Delta m_{t}\right)$ provides a test of CPT invariance. CMS measures $\Delta m_{t}$ using $19 \mathrm{fb}^{-1}$ of 8 $\mathrm{TeV}$ data collected by the CMS experiment with the result $\Delta m_{t}=272 \pm 196$ (stat.) \pm 122 (syst.) $\mathrm{MeV}$ that is consistent with the standard model prediction [18]. The accuracy of this result is significantly higher than that of existing measurements. In figure 7, the measured values of $m_{t}$ for events containing a negatively charged lepton and a positively charged lepton are shown separately.
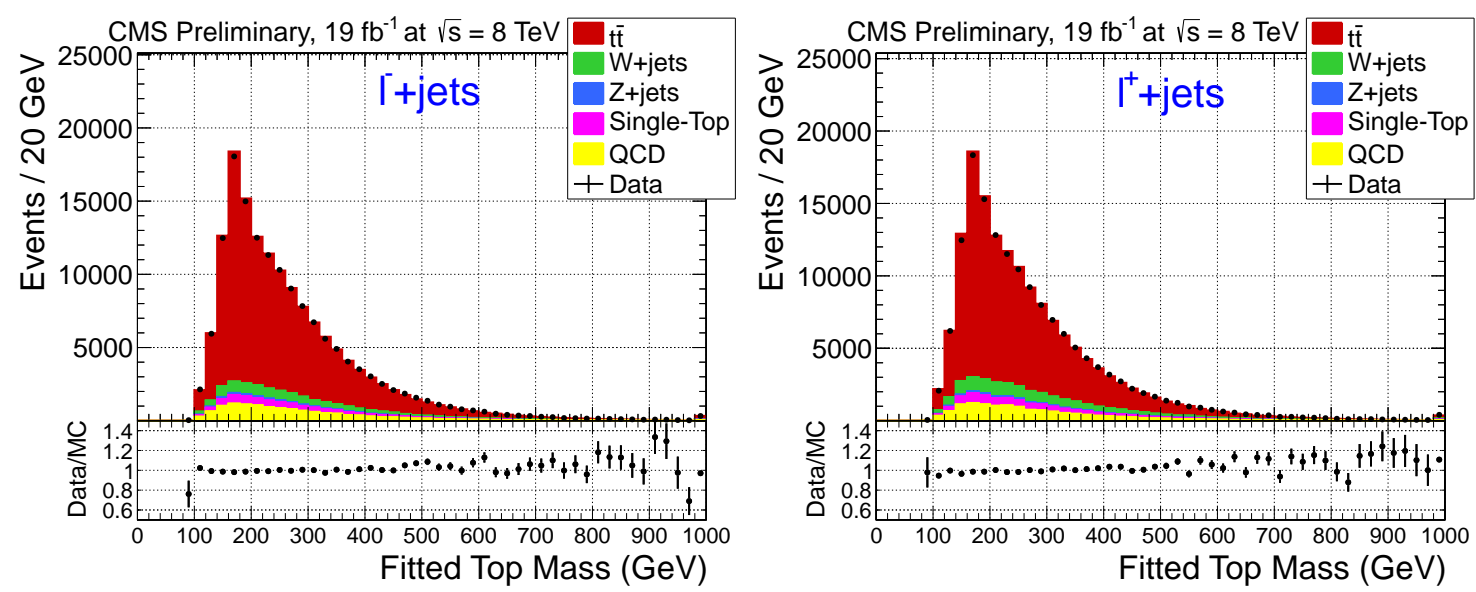

Figure 7: The measured values $m_{t}$ for events containing a negatively charged lepton (left) and a positively charged lepton (right) are shown.

\section{Measurements of the quark properties}

In this section a selection of the CMS measurements of top-quark properties are detailed. Details of results concerning the top-quark polarisation, spin correlations and charge asymmetries in $t \bar{t}$ events not discussed here may be found in [19], [20], [21] and [22].

\subsection{Search for FCNC in top-quark decay}

CMS performs a search for flavour-changing neutral currents (FCNC) in top-quark decays [23]. The analysis focuses on the decay chain $t \bar{t} \rightarrow Z q+W b$ with both $\mathrm{W}$ and $\mathrm{Z}$ decaying leptonically producing three leptons (electrons or muons) in the final state. The dataset used corresponds to an integrated luminosity of $19.7 \mathrm{fb}^{-1}$ at $\sqrt{s}=8 \mathrm{TeV}$. In figure 8 , the invariant mass distributions of kinematically reconstructed $\mathrm{Zj}$ and $\mathrm{Wb}$ systems are shown. No excess of events over the standard model expectation is observed. Hence upper limits on the branching ratio of $t \rightarrow \mathrm{Zq}$ of $0.05 \%$ at $95 \%$ confidence level are calculated.

\subsection{W polarisation}

CMS performs a measurement of the $\mathrm{W}$ boson helicity fractions in $t \bar{t}$ events with $4.6 \mathrm{fb}^{-1}$ of data with $\sqrt{s}=7 \mathrm{TeV}$ [24]. The analysis is based on the dileptonic (electron or muon) decay channel of the $t \bar{t}$ system. In order to kinematically reconstruct the $t \bar{t}$ system in events containing two leptons an analytic method developed within the CMS collaboration is applied [27], [28]. The 

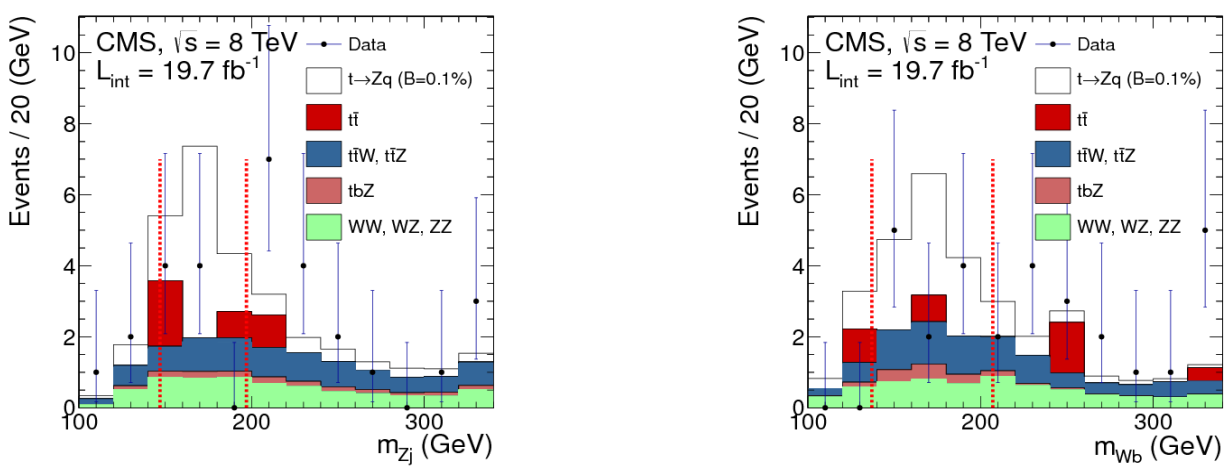

Figure 8: The invariant mass distributions of the kinematically reconstructed $\mathrm{Zj}$ and $\mathrm{Wb}$ systems are shown.

reconstructed system produced by this method are used to calculate the helicity angle $\left(\cos \theta^{*}\right)$. $\cos \theta^{*}$ is defined as the angle between the charged lepton three-momentum in the $\mathrm{W}$ rest frame and the $\mathrm{W}$ momentum in the top-quark rest frame. In figure 9 the $\cos \theta^{*}$ distributions for data and simulation are shown. The fractions of left-handed $\left(F_{L}\right)$, longitudinal $\left(F_{0}\right)$ and right-handed $\left(F_{R}\right)$ W bosons are extracted from a fit to this distribution with the result: $F_{L}=0.288 \pm 0.035$ (stat) \pm 0.040 (sys), $F_{0}=0.698 \pm 0.057$ (stat) \pm 0.063 (sys) and $F_{R}=0.014 \pm 0.027$ (stat) $\pm 0.042($ sys $)$. This result is consistent with the standard model expectation. CMS also measures the $\mathrm{W}$ boson helicity fractions in single top-quark production [25]. Combined results produced by the CMS and ATLAS experiments are also available [26].

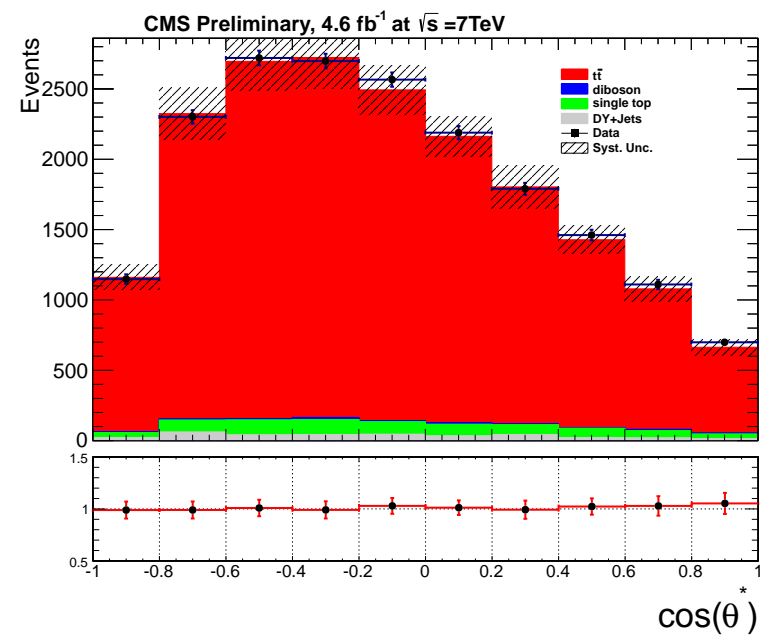

Figure 9: The $\cos \theta^{*}$ distribution for data and simulation is shown.

\section{Conclusion}

In this note a selection of results from the CMS top-quark physics program using datasets with centre-of-mass energies of 7 and $8 \mathrm{TeV}$ recorded by CMS in the years 2010 to 2012 have been presented. The results concern the measurement of the cross sections of processes involving $t \bar{t}$ 
systems, measurements of the top-quark mass and measurements of the top-quark properties. All results are consistent with the expectations of the standard model.

\section{References}

[1] Study of single top-quark production with the CMS detector, talk given at QFTHEP 2013, the XXI International Workshop High Energy Physics and Quantum Field Theory, June 23 - June 30, 2013 Saint Petersburg Area, Russia.

[2] The CMS top quark physics results public webpage, https://twiki.cern.ch/twiki/bin/view/CMSPublic/PhysicsResultsTOP

[3] Top pair cross section in e/mu+jets at $8 \mathrm{TeV}$, The CMS Collaboration, CMS-PAS-TOP-12-006 (2012).

[4] Cross section measurement in the di-lepton channel at $8 \mathrm{TeV}$, The CMS Collaboration, CMS-PAS-TOP-12-007, submitted to J. High Energy Phys, arXiv:1312.7582 (2012).

[5] Measurement of the differential ttbar cross section in the dilepton channel at $8 \mathrm{TeV}$, The CMS Collaboration, CMS-PAS-TOP-12-028 (2012).

[6] Measurement of the differential ttbar cross section in the lepton+jets channel at $8 \mathrm{TeV}$, The CMS Collaboration, CMS-PAS-TOP-12-027 (2013).

[7] Measurement of differential top-quark-pair production cross sections in pp collisions at $\sqrt{s}=7 \mathrm{TeV}$, The CMS Collaboration, Eur. Phys. J. C (2013) 73:2339.

[8] Measurement of the Jet Multiplicity in Di-leptonic Top Quark Pair events at 8 TeV, The CMS Collaboration, CMS-PAS-TOP-12-041 (2013).

[9] Measurement of jet multiplicity top pair events, The CMS Collaboration, CMS-PAS-TOP-12-018 (2012).

[10] Measurement of $\sigma(t t b b) / \sigma(t t j j)$ at 7TeV, The CMS Collaboration, CMS-PAS-TOP-12-24 (2012).

[11] Measurement of associated production of vector bosons and top quark-antiquark pairs in pp collisions at $\sqrt{s}=7 \mathrm{TeV}$, The CMS Collaboration, Phys. Rev. Lett. 110, 172002 (2013).

[12] Measurement of the top-quark mass in $t \bar{t}$ events with lepton+jets final states in pp collisions at $\sqrt{s}=7$ $\mathrm{TeV}$, The CMS Collaboration, J. High Energy Phys 12(2012) 105.

[13] Study of the dependence of the top-quark mass measurement on event kinematics, The CMS Collaboration, CMS-PAS-TOP-12-029 (2012).

[14] MadGraph 5 : Going Beyond, Alwall, Johan and Herquet, Michel and Maltoni, Fabio and Mattelaer, Olivier and Stelzer, Tim, J. High Energy Phys 1106128 (2011).

[15] A New method for combining NLO QCD with shower Monte Carlo algorithms Nason, Paolo, J. High Energy Phys. 0411040 (2004).

[16] Matching NLO QCD computations with Parton Shower simulations: the POWHEG method S. Alioli, P. Nason, C. Oleari and E. Re, J. High Energy Phys. 1006 (2010) 043 [arXiv:1002.2581 [hep-ph]]

[17] Matching NLO QCD computations and parton shower simulations S. Frixione and B.R. Webber, J. High Energy Phys. 0206 (2002) 029 [hep-ph/0204244]

[18] Measurement of the top/antitop mass difference, The CMS Collaboration, CMS-PAS-TOP-12-031 (2012). 
[19] Top polarisation with the di-lepton channel, The CMS Collaboration, CMS-PAS-TOP-12-016 (2012).

[20] Top spin correlations in the di-lepton channel, The CMS Collaboration, CMS-PAS-TOP-12-004 (2012).

[21] Top charge asymmetry measurement in dileptons at $7 \mathrm{TeV}$, The CMS Collaboration, CMS-PAS-TOP-12-010 (2012).

[22] Measurement of the ttbar charge asymmetry with lepton+jets events at $8 \mathrm{TeV}$, The CMS Collaboration, CMS-PAS-TOP-12-033 (2013).

[23] Search for flavor changing neutral currents in top quark decays in proton-proton collisions at $8 \mathrm{TeV}$, The CMS Collaboration, CMS-PAS-TOP-12-037 (2012).

[24] Measurement of W-Polarization in Di-Leptonic ttbar Events in pp Collisions with $\sqrt{s}=7 \mathrm{TeV}$, The CMS Collaboration, CMS-PAS-TOP-12-015 (2012).

[25] Measurement of W-helicity fractions in single top events topology, The CMS Collaboration, CMS-PAS-TOP-12-020 (2013).

[26] Measurement of W-helicity fractions in top decays (LHC combination), The CMS Collaboration, CMS-PAS-TOP-12-025 (2013).

[27] Algebraic approach to solve t $\bar{t}$ dilepton equations, L. Sonnenschein, Phys.Rev. D72 (2005) 095020, doi:10.1103/PhysRevD.72.095020, arXiv:hep-ph/0510100

[28] Analytical solution of ttbar dilepton equations, L. Sonnenschein, Phys.Rev. D73 (2006) 054015, doi:10.1103/PhysRevD.73.054015,10.1103/PhysRevD.78.079902, arXiv:hep-ph/0603011. 